\title{
Transient Splenial Lesion of the Corpus Callosum Related to Migraine with Aura in a Pediatric Patient
}

\author{
Olcay Ünver, MD ${ }^{1, *}$, Büşra Kutlubay, MD ${ }^{1}$, Tolga Besci, MD', Gazanfer Ekinci, MD ${ }^{3}$, \\ Feyyaz Baltacıŏ̆lu, $\mathrm{MD}^{3}$, Dilşad Türkdoğan, $M^{3}$
}

\author{
${ }^{1}$ Department of Pediatric Neurology, Marmara University, İstanbul, Turkey \\ ${ }^{2}$ Department of Pediatrics, Marmara University, İstanbul, Turkey \\ ${ }^{3}$ Department of Radiology, Marmara University, İstanbul, Turkey \\ * Corresponding author: Marmara University Fevzi Çakmak Mah. Muhsin Yazıcıoğlu Cad. No: 10 Üst, Kaynarca / Pendik / \\ İstanbul, Turkey; e-mail: olcaymd@hotmail.com
}

\begin{abstract}
Summary: Background: Transient splenial lesions of the corpus callosum are rare radiological findings first described in association with epilepsy, antiepileptic drugs and viral encephalitis. However, subsequently more cases were described associated with diverse clinical conditions. Case report: We describe a 13-year-old girl suffering from migraine with aura presenting with headache, right-sided hemiparesis and encephalopathy. Brain magnetic resonance imaging revealed an ovoid lesion in the splenium of the corpus callosum. The patient's neurological symptoms resolved within 3 days without therapy and the lesion disappeared in follow up magnetic resonance images obtained 3 weeks after the onset of the symptoms. Results: Migraine with aura was considered to be the cause of the lesion. To our knowledge the present case is the first report of a pediatric patient with a diagnosis of migraine with aura presenting with hemiparesis and encephalopathy. Conclusions: A diagnosis of transient lesion of the corpus callosum should be suspected in patients with migraine with aura presenting with hemiparesis and encephalopathy. A mild course and a good prognosis might be expected in the presence of a splenial lesion of the corpus callosum.
\end{abstract}

Keywords: Transient splenial lesion; Corpus callosum; Migraine with aura; Encephalopathy; Hemiparesis

\section{Introduction}

Transient splenial lesions of the corpus callosum are described in association with many diverse clinical conditions including various infections, use or withdrawal of antiepileptic drugs, and hypoglycemia (1-6). The lesion appears as a well-defined hyperintense ovoid lesion in the center of the splenium of the corpus callosum, best observed in diffusion weighted brain magnetic resonance images. The pathology is unclear as well as the specific location of the lesion. This new clinico-radiological entity presents with mild encephalitis/encephalopathy in clinical practice (2). The usual clinical manifestations include disturbance of consciousness, delirium, seizures and headache, which resolve in a couple of days. The lesion itself also disappears in follow-up radiological images $(1,2)$.

Here we present a pediatric case with a transient splenial lesion of the corpus callosum related to migraine with aura. To our knowledge our patient is the first pediatric case of transient splenial lesion of the corpus callosum related to migraine with aura presenting with encephalopathy and hemiparesis.

\section{Case report}

A 13-year old girl was admitted with a severe headache, confusion, violent behavior, slurred speech, and right-sided hemiparesis. She first experienced similar but milder attacks of a throbbing headache accompanied by nausea and vomiting following numbness feeling in the lips and slurred speech, which had lasted for 2-3 hours and not complicated with hemiparesis, two months before. At the time, brain magnetic resonance imaging (MRI) and electroencephalography (EEG) were unremarkable. After checking family history it was learned that her mother and her aunt suffer from migraine and the diagnosis of migraine with aura was made according to the criteria of International Classification of Headache Disorders two months before (7). Her parents stated that this last attack was the worst one and the symptoms lasted longer and complicated with loss of muscle strength, personality changes and violent behavior. On admission she was confused. On physical examination she was afebrile. Neurological examination was otherwise unremarkable except for 4/5 muscle strength on the right side of her body. Routine laboratory tests including complete blood count, biochemistry, electrolytes, prothrombin time and partial 

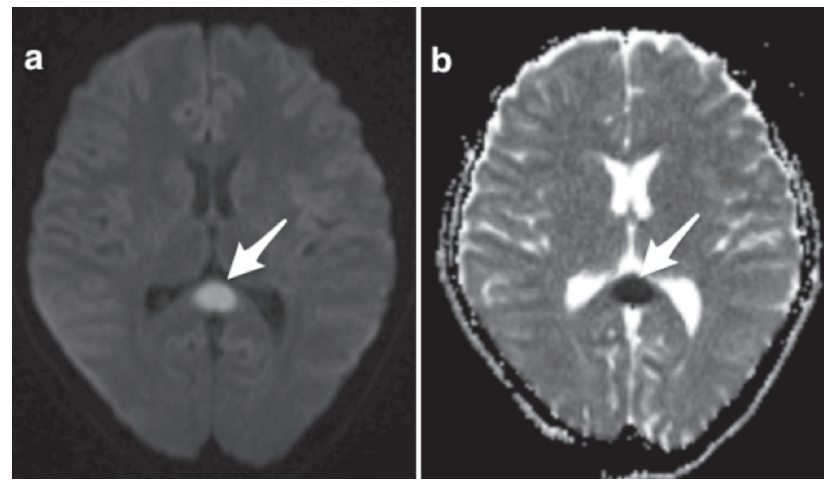

Fig. 1: Transient splenial lesion in a pediatric patient suffering from migraine with aura. An axial diffusion weighted magnetic resonance image shows a hyperintense lesion in the splenium of the corpus callosum (a) and corresponding hypointense lesion in apparent diffusion coefficient images (b).
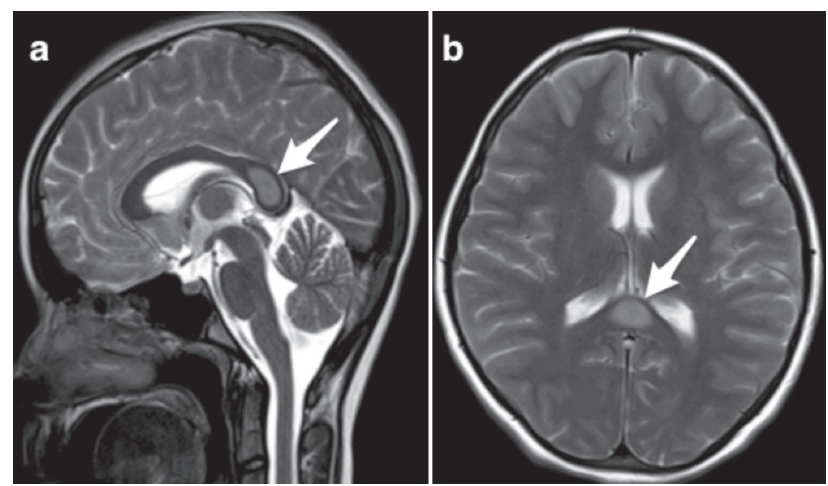

Fig. 2: Transient splenial lesion in a pediatric patient suffering from migraine with aura in a sagittal T2 weighted image (a) and in an axial T2 weighted image (b).

thromboplastin time were all normal. C-reactive protein and erythrocyte sedimentation rate were within normal limits. Serum antibody titers to Epstein-Barr virus, Cytomegalovirus, Hepatitis $B$ and $C$ virus and Varicella Zoster virus, Parvovirus B19, Mycoplasma pneumonia, Legionella pneumophila, Chlamydia pneumonia were all normal. A lumbar puncture revealed a normal opening pressure, a normal leucocyte count and normal glucose and protein levels. Brain MRI conducted that day revealed a well-circumscribed ovoid lesion in the splenium of the corpus callosum, hyperintense in diffusion-weighted magnetic resonance images and hypointense in apparent diffusion coefficient images (Figure 1a,b). The lesion exhibited moderate hyperintensity in T2 weighted images (Figure 2a,b). No enhancement was evident after gadolinium administration. Her EEG revealed generalized, high amplitude intermittent slow delta waves prominent on frontal areas, which is consistent with encephalopathy (Figure 3a). Neurological examination revealed normal results about 36 hours after the first neurological symptoms. Follow-up EEG on day 3 showed significant
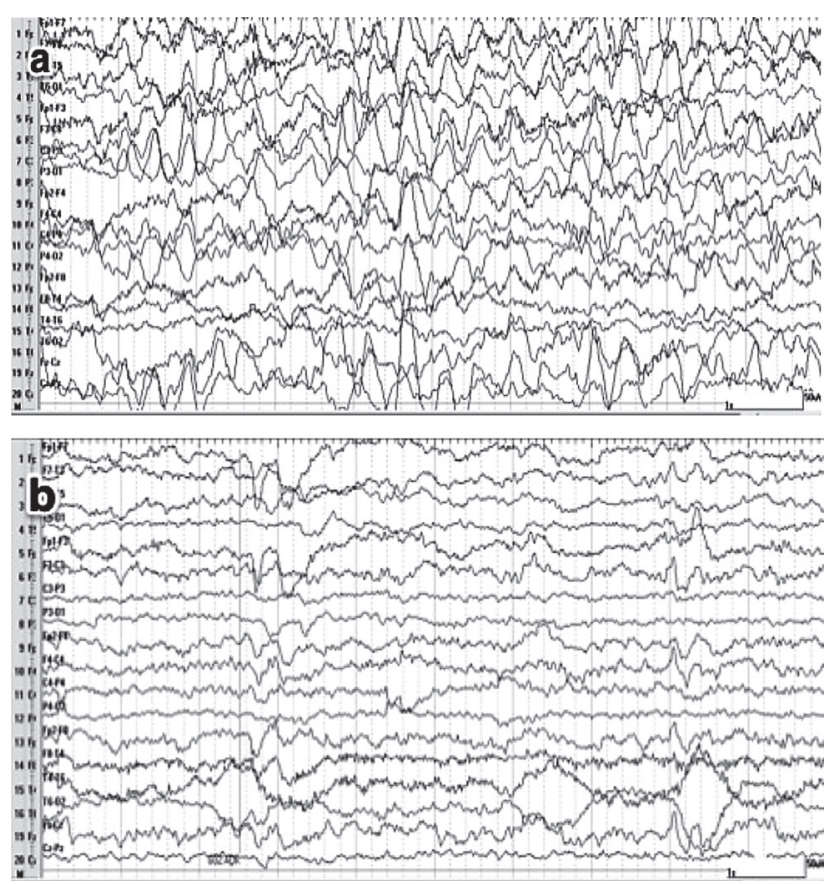

Fig. 3: Electroencephalography of the patient. Global diffuse waves prominent on the frontal areas consistant with encephalopathy (a). İmprovement of EEG after 3 days (b).

improvement (Figure 3b). She was discharged 4 days after admission with a completely normal neurological examination. Her follow-up MRI 3 weeks after the first MRI, revealed a complete disappearance of the lesion.

\section{Discussion}

We reported an unusual case of transient splenial lesion of the corpus callosum presenting with encephalopathy and right-sided hemiparesis related to migraine with aura in a pediatric patient. In recent years, a uniform temporary lesion confined only to the splenium of the corpus callosum has been repeatedly reported $(1,3,8)$. The typical presentation is a well defined ovoid lesion in the center of the corpus callosum, which is hyperintense on T2 weighted images iso- or hypointense on T1 weighted images with no evidence of contrast enhancement and best observed on diffusion weighted images with low apparent diffusion coefficient values, which indicates restricted diffusion (9). To our knowledge 2 cases of migraine with aura as an associated condition have been reported to date. One of them is an adult patient with sensory aura (9) and the other a 17-year old girl with acephalgic migraine with a visual aura triggered by stimulant containing diet pills (10). None of these two patients presented with encephalitis or hemiparesis.

In a study where 5 patients with transient splenial lesion of the corpus callosum associated with influenza virus infection were presented, one patient had motor deficits explained by additional white matter lesions (3). In another report, an 
adult case related to adenovirus infection had right hemiparesis and hemianesthesia; however, no white matter lesions were detected on MRI images (4). The authors have two hypotheses; either a culprit lesion located within the deep white matter above the midbrain, which is not detectable on MRI images or the splenial lesion itself is responsible for the motor and sensory deficit. The authors support the first hypothesis based on the neuroanatomical localization of the lesion; however, they also state that the second option cannot be ruled out entirely, because of a previous report, which described corpus callosum body as being responsible for hemiparesis (11).

The exact pathology of the lesion is still unclear. Until today, many hypotheses have been described to explain the pathophysiology of the splenial lesion such as reversible demyelination probably due to antiepileptic drug toxicity or abrupt stoppage of chronic antiepileptic therapy, which could lead to ischemia and resultant cytotoxic edema $(12,13)$. Additionally, extrapontine osmotic myelinolysis due to sodium and glucose imbalance, and direct viral invasion have been held responsible for the lesion (14). However, most of the authors support that intramyelinic edema from the separation of myelin layers and inflammatory infiltrate rather than a breakdown of the blood brain barrier or demyelination are responsible for the lesion $(1,3)$, which explains the reversibility of the diffusion restriction of the lesion. Similarly, the reason for the specific predilection for the splenium of the corpus callosum has not been clarified yet (3).

All the patients' symptoms resolve invariably in a couple of days and the lesion disappears in follow-up MRI studies performed from 3 days to 3 months (15). To our knowledge, the lesion was detected only in 1 case in $\mathrm{T} 2$ weighted images over 5 months although decreased in size indicating that it could result in gliosis (15).

\section{Conclusion}

Clinicians should consider transient splenial lesion of corpus callosum in children with known migraine present- ing with headache, encephalopathy and hemiparesis. A mild course and a good prognosis might be expected based on the reports to date.

\section{References}

1. Tada H, Takanashi J, Barkovich AJ, et al. Clinically mild encephalitis/encephalopathy with a reversible splenial lesion. Neurology 2004; 63:1854-8.

2. Takanashi J. Two newly proposed infectious encephalitis/encephalopathy syndromes. Brain Dev 2009; 31: 521-8.

3. Bulakbaşı N, Kocaoğlu, Tayfun C, Uçoz T. Transient splenial lesion of the corpus callosum in clinically mild influenza associated encephalitis/encephalopathy. Am J Neuroradiol 2006; 27: 1983-6.

4. Hibino M, Horiuchi S, Okubo Y, Kakutani T, Ohe M, Kondo T. Transient hemiparesis and hemianesthesia in an atypical case of adult-onset clinically mild encephalitis/encephalopathy with a reversible splenial lesion associated with adenovirus infection. Intern Med 2014; 53: 1183-5.

5. Meleková A, Andrlová L, Král P, Ungermann L, Ehler E. Encephalitis with Prolonged but Reversible Splenial Lesion. Acta Medica (Hradec Králové) 2015; 58(3): $108-12$.

6. Degirmenci E, Degirmenci T, Cetin EN, Kıroğlu Y. Mild encephalitis/encephalopathy with a reversible splenial lesion (MERS) in a patient presenting with papilledema. Acta Neurol Belg. 2015 Jun; 115(2): 153-5.

7. Headache Classification Subcommitte of the International Headache Society. The International classification of headache disorders: 2nd edition. Cephalgia 2004; 24(suppl 1): 9-160.

8. Matsubara K, Kodera M, Nigami H, Yura K, Fukaya T. Reversible lesion in influenza virus encephalopathy. Pediatr Neurol 2007; 37: 431-4.

9. Yang CY, Lin FY. Reversible splenial lesion of the corpus callosum in migraine with aura. The Neurologist 2011; 17: 157-9.

10. Wislow H, Mickey B, Frohman EM. Sympathomimetic-induced kaleidoscopic visual illusion associated with a reversible splenium. Arch Neurol 2006; 63: $135-7$.

11. Jang SH, Lee J, Yeo SS, Chang MC. Callosal disconnection syndrome after corpus callosum infarct: a diffusion tensor tractography study. J Stroke Cerebrovasc Dis 2013; 22: e240-e4.

12. Kim SS, Chang KH, Kim ST, et al. Focal lesion in the splenium of the corpus callosum in epileptic patients: Antiepileptic drug toxicity? Am J Neuroradiol 1999; 20: $125-9$.

13. Gürtler S, Ebner A, Tuxhorn I, Ollech I, Pohlmann-Eden B, Woermann FG. Transient lesion in the splenium of the corpus callosum and antiepileptic drug withdrawal Neurology 2005; 65: 1032-6.

14. Polster T, Hoppe M, Ebner A. Transient lesion in the splenium of corpus callosum: three further cases in epileptic patients and a pathophysiological hypothesis. J Neurol Neurosurg Psychiatry 2001; 70: 459-463.

15. Hashimoto Y, Takanashi J, Kaiho K, et al. A splenial lesion with transiently reduced diffusion in clinically mild encephalitis is not always reversible. A case report. Brain Dev 2009; 31: 710-2.

Received: 03/03/2016 Accepted: 10/05/2016 